\title{
Mortalidade por AIDS no Estado do Rio de Janeiro - 1991 a 1995
}

\author{
AIDS mortality in the State \\ of Rio de Janeiro, 1991-1995
}

Katia Regina Valente de Lemos 1

Joaquim Gonçalves Valente 2

1 Secretaria de Estado de Saúde do Rio de Janeiro. Rua México 128, sala 416, Rio de Janeiro, $R J$ 20031-142, Brasil. 2 Instituto de Medicina Social, Universidade do Estado do Rio de Janeiro. Rua São Francisco Xavier 524, 7 o andar, Rio de Janeiro, $R J$ 20559-990, Brasil.

\begin{abstract}
This article focuses on the AIDS mortality profile as related to socioeconomic and geographic variables, as well as evaluating the impact of deaths from AIDS in the State of Rio de Janeiro. The analysis included all death certificates for residents of the State from 1991-1995, 10,024 of which had AIDS recorded as the primary cause of death. In the 20-49-year age bracket, among individuals who had died of AIDS, the proportion of those with university schooling (14\%) and the proportion of single individuals (75\%) were greater than the respective proportions for all other causes of death (5.4\% and 56.3\%). For the population as a whole, the AIDS mortality rate increased from 1991 to 1995, from 20.6/100,000 to 30.2/100,000 for males and from 3.7/100,000 to 7.9/100,000 for females. For the year 1995 in the 20-49-year bracket, considering the 17 groups of causes from the ICD-9, AIDS was the third most common cause of death among men and the fifth most common among women. The proportion of Potential Loss of Life Years up to 65 years as a function of AIDS increased from 1991 to 1995, from 3.4\% to 4.7\% for men and from $1.4 \%$ to $2.9 \%$ for women. During this same period there was also an increase in the number of counties in the State of Rio de Janeiro with reported deaths from AIDS.
\end{abstract}

Key words Acquired Immunodeficiency Syndrome; Mortality; Death Certificates

Resumo O estudo objetivou conhecer o perfil dos óbitos por AIDS segundo variáveis sócio-econômicas e geográficas e avaliar o impacto da mortalidade por essa causa no Estado do Rio de Janeiro. A análise considerou todas as declarações de óbito de residentes no Estado, ocorridas no período de 1991 a 1995, das quais 10.024 tiveram como causa básica a AIDS. Verificou-se que no grupo entre 20 e 49 anos, a proporção de indivíduos com nível universitário (14\%) e de solteiros (75\%) entre os óbitos por AIDS foi maior que a observada entre os óbitos pelas demais causas, respectivamente 5,4\% e 56,3\%. No total da população a taxa de mortalidade por AIDS cresceu de 1991 para 1995, passando nos homens de 20,6/100.000 para 30,2/100.000 e nas mulheres de 3,7/100.000 para 7,9/100.000. No ano de 1995 e na população de 20 a 49 anos, considerando-se os 17 grupos de causas da CID-9, a AIDS constituiu-se na terceira causa de morte entre os homens e na quinta causa entre as mulheres. A proporção de Anos Potenciais de Vida Perdidos até 65 anos em função da AIDS cresceu de 1991 para 1995, passando nos homens de 3,4\% para 4,7\% e, nas mulheres, de 1,4\% para 2,9\%. Observou-se o aumento do número de municípios com óbitos por AIDS.

Palavras-chave Síndrome de Imunodeficiência Adquirida; Mortalidade; Atestado de Óbito 


\section{Introdução}

A Síndrome da Imunodeficiência Adquirida (AIDS) pode ser considerada como um relevante problema de saúde enfrentado a partir da década de 80, caracterizando-se, no que tange à mortalidade, por sua crescente participação entre as principais causas de morte, particularmente, entre os adultos jovens.

Durante os anos 80, a infecção pelo HIV emergiu como uma das principais causas de morte nos Estados Unidos entre homens de 25 a 44 anos. Neste sexo e grupo etário, foi, em 1990, a principal causa de morte em 64 cidades americanas (Selik et al., 1993). Em 1994, estimou-se que 41.930 pessoas residentes nos Estados Unidos morreram devido à infecção pelo HIV, das quais $72 \%$ tinham entre 25 e 44 anos. Neste mesmo ano, a infecção pelo HIV ocupou, entre 72 causas selecionadas pelo Centers for Disease Control and Prevention (CDC) dos Estados Unidos, a oitava posição, constituindose, na população de 25 a 44 anos, na principal causa de morte entre os homens e na terceira causa entre as mulheres (CDC, 1996b).

Também na Europa, a AIDS tem despontado como importante causa de morte. Em cidades como Paris e Nice, a AIDS respondeu, para a população entre 25 e 44 anos, por respectivamente $40 \%$ e $44 \%$ dos óbitos ocorridos entre os homens e por $13 \%$ e $24 \%$ dos óbitos ocorridos entre as mulheres (Bouvier-Colle et al., 1994). Na Itália, um estudo que utilizou razões de mortalidade padronizada (RMP) para os anos de 1980, 1985 e 1990 comparada com a RMP em 1975, mostrou que, para 1990, o excesso de mortalidade encontrado nos homens entre 20 e 35 anos, com RMP > 130 foi inteiramente atribuível à AIDS (La Vecchia et al., 1994). A Espanha tem, desde 1990, a incidência anual de AIDS mais alta da Europa, com uma taxa de mortalidade que passou, entre 1985 e 1991, de 0,3 para 25,5/100.000 no grupo de idade entre 25 e 34 anos e de 0,3 para 9,9/100.000, no grupo de 35 a 44 anos (Regidor et al., 1995). Em Amsterdam, Holanda, um estudo sobre o impacto da mortalidade por AIDS no período de 1982 a 1992 mostrou que, nesta cidade, a AIDS tornou-se a primeira causa de morte entre homens de 25 a 54 anos a partir de 1990 (Bindels et al., 1994).

Em muitas cidades africanas, a infecção pelo HIV tem-se constituído na primeira causa de morte na população adulta. Em Kigali, Ruanda, por exemplo, onde $30 \%$ dos adultos estavam soropositivos para o HIV em 1988, a infecção pelo HIV esteve associada com $90 \%$ das mortes em mulheres em idade fértil (Perre, 1995).
Poucos estudos referem-se à mortalidade por AIDS em países da América Latina, mas segundo dados do Joint United Nations Programme on HIVIAIDS (UNAIDS, 1997), nesta região como um todo, a AIDS já ultrapassava, em 1997, os acidentes de trânsito como causa de morte. No México, a AIDS passou a constituir-se em importante causa de morte a partir de 1987 com uma taxa de 3,6/100.000 que passou, em 1992, para 29,4/100.000 (ValdespinoGómez et al., 1995).

Por atingir principalmente uma população jovem, a AIDS é também responsável por grande número de Anos Potenciais de Vida Perdidos (APVP). Nos Estados Unidos, em 1994, a infecção pelo HIV respondeu por $9 \%$ dos APVP por todas as causas antes dos 65 anos de idade (APVP-65), tornando-se a quarta causa de APVP65 (CDC, 1996b). Na Inglaterra e País de Gales, em 1991, as mortes por AIDS foram responsáveis por $2 \%$ dos APVP-65 em homens. Na Austrália, em 1988, a AIDS resultou em mais anos perdidos por mortes prematuras em homens que o diabetes mellitus ou melanoma cutâneo, e, em 1991, foi uma causa de morte prematura mais freqüente que as doenças cerebrovasculares (Gold et al., 1994). A AIDS constituiu-se também em uma das primeiras causas de APVP na Espanha, notadamente na região de Catalunha e na cidade de Barcelona (Montellá-i-Jordana et al., 1995; Mur et al., 1995).

No Brasil, os dados disponíveis no Sistema de Informação sobre Mortalidade (SIM) (MS, 1997b) mostram que, apenas em 1995, 15.156 brasileiros morreram em conseqüência da AIDS. Destes, $13.205(87,1 \%)$ tinham entre 20 e 49 anos de idade. Nesta faixa etária, a AIDS ocupou, em 1995, o quinto lugar, se comparada com todas as outras causas de morte agregadas segundo os grandes capítulos da Classificação Internacional de Doenças - Nona Revisão (CID-9) (OMS, 1985). Estudos referentes ao Estado de São Paulo revelam que a AIDS vem modificando o padrão de mortalidade no Estado, constituindo-se em importante causa de morte entre os adultos jovens (Ferreira \& Castiñeiras, 1996; Waldvogel, 1994). Na capital paulista, conforme dados do Boletim Epidemiológico da Secretaria Municipal de Saúde de São Paulo (SMS-SP, 1997), desde 1993, a AIDS tornou-se a primeira causa de morte entre as mulheres de 15 a 49 anos, sendo precedida nos homens da mesma faixa etária, apenas pelos homicídios, desde 1991. Apesar disso, observou-se queda no total de óbitos a partir de 1996.

A realização do presente estudo teve como base a importância da AIDS como causa de morte, aliada à escassez de estudos sobre o te- 
ma no Brasil e, sobretudo, no Estado do Rio de Janeiro. Pretende-se com este trabalho, além de avaliar o impacto da mortalidade por AIDS no Estado, conhecer o perfil dos óbitos por AIDS segundo variáveis sócio-econômicas e geográficas.

\section{Material e método}

Foram analisadas 556.342 declarações de óbitos de residentes no Estado do Rio de Janeiro, ocorridas no período de 1991 a 1995, das quais 10.024 tiveram como causa básica a AIDS.

\section{Fonte de dados}

Os dados referentes às declarações de óbitos foram obtidos valendo-se do SIM, cedido pela Secretaria de Estado de Saúde do Rio de Janeiro (SES-RJ). Cabe, portanto, esclarecer que no processamento dos óbitos no nível estadual não estavam ainda incorporados os óbitos de residentes no Estado do Rio de Janeiro ocorridos em outras unidades da federação. No entanto, a divulgação pelo Ministério da Saúde (MS) dos dados nacionais, em julho de 1997, permitiu verificar que no Estado do Rio de Janeiro, menos de $1 \%$ das mortes por todas as causas e especificamente por AIDS ocorreram fora do Estado.

Os dados relativos às populações de $1992 \mathrm{a}$ 1995 foram cedidos pelo MS, que adotou a seguinte metodologia: o IBGE (Fundação Instituto Brasileiro de Geografia e Estatística) forneceu ao MS a estimativa por extrapolação geométrica, com base nos Censos Geográficos de 1980 e 1991, da população total dos municípios, sem discriminação de sexo e faixa etária. O MS aplicou a estas populações a distribuição percentual por sexo e faixa etária obtidas do Censo Demográfico de 1991. Para o ano de 1991, foram utilizados os dados censitários, corrigidos para primeiro de julho pelo Centro de Desenvolvimento e Planejamento Regional (CEDEPLAR) da Universidade Federal de Minas Gerais.

Com a finalidade de estabelecer comparações no tempo, foram obtidas informações sobre a mortalidade por AIDS referente ao período entre 1984 (ano em que aparecem os primeiros óbitos por AIDS) e 1990, anterior ao período de estudo, tendo-se utilizado dados do SIM do MS e dados de população fornecidos pelo CEDEPLAR.

\section{Tratamento dos dados}

Foram selecionadas as variáveis: ano de ocorrência do óbito, sexo, idade, causa básica de óbito, município de residência, microrregião e mesorregião geográfica, grau de instrução e estado civil.

Para diminuir os erros de registro, foram comparadas a idade registrada e a idade calculada a partir do ano de nascimento e do ano do óbito. Quando as idades diferiram em mais de dois anos, considerou-se a idade como ignorada. Os óbitos foram agrupados em quatro grandes grupos etários (menor de 13 anos, 13 a 19, 20 a 49 e 50 e mais) e em faixas etárias com intervalos de cinco anos até a idade de 69 anos, sendo as idades posteriores agrupadas em "70 anos e mais". Considerou-se como adultos os indivíduos com 13 anos de idade ou mais e como crianças os indivíduos com menos de 13 anos. Esta classificação objetivou compatibilização com aquela utilizada pelo MS na definição do caso de AIDS.

A codificação do óbito é referente à nona revisão da CID-9. Os óbitos foram agrupados em grandes grupos de causas, que compõem os dezessete capítulos da CID-9 (OMS, 1985). O capítulo III foi subdividido em III (excluídos os óbitos por AIDS) e IIIa (contendo somente os óbitos por AIDS). Os óbitos foram agrupados também de acordo com a lista de cinqüenta causas para a mortalidade da CID-9, incluindo-se nela uma rubrica para a AIDS.

Devido aos problemas operacionais resultantes das mudanças na divisão municipal do estado no período do estudo, optou-se por manter a distribuição geográfica de 1991 com um total de 70 municípios. Assim, tanto os óbitos quanto as populações dos municípios emancipados a partir de 1992 foram incorporados aos seus municípios de origem, agrupados, posteriormente, segundo as microrregiões e mesorregiões geográficas definidas pelo IBGE.

A variável "grau de instrução" obedeceu à classificação disponível na declaração de óbito: nenhuma (analfabeto), fundamental (primeiro grau completo ou incompleto), segundo grau (completo ou incompleto), superior (universitário completo ou incompleto) e ignorado.

Da mesma forma que para "grau de instrução", a variável "estado civil” foi classificada de acordo com as informações disponíveis na declaração de óbito: solteiro, casado, viúvo, desquitado ou divorciado, outro e ignorado. 


\section{Análise dos dados}

Compararam-se as médias de idade e a distribuição proporcional segundo escolaridade, estado civil e área geográfica de residência dos óbitos por AIDS com a dos óbitos pelas demais causas. A fim de evitar confundimento por idade, já que, nos óbitos pelas demais causas os indivíduos são mais velhos e objetivando retirar o efeito de coorte, visto que, os indivíduos mais velhos têm menor nível de instrução, as comparações relativas à escolaridade e ao estado civil foram restritas à faixa etária entre $20 \mathrm{e}$ 49 anos.

Foram estimados os indicadores de mortalidade proporcional segundo sexo, faixa etária e ano de ocorrência e as taxas de mortalidade específicas por AIDS segundo as mesmas variáveis, além do município de residência. Os indicadores de mortalidade proporcional e as taxas de mortalidade foram calculados ano a ano a fim de se observarem possíveis modificações da mortalidade por AIDS durante o período de estudo.

Foram calculados os APVP-65, tendo como pressuposto a distribuição uniforme das mortes, supondo a ocorrência das mesmas na metade do intervalo de cada grupo etário, com exceção dos menores de um ano de idade. Neste grupo etário, em função de a maior parte das mortes ocorrerem no início do intervalo (Kuzma, 1984), foi utilizado como ponto de corte um quarto do período (0,25 anos).

O software utilizado para a análise dos dados foi o Epi Info versão 6.03 (CDC/WHO, 1996). Para a construção dos cartogramas foi utilizado o software MapInfo versão 5.0 (MapInfo Corporation, 1994).

\section{Resultados}

\section{Perfil dos óbitos por AIDS}

A distribuição dos óbitos por AIDS segundo sexo mostrou uma proporção de $80,1 \%$ de homens e $19,9 \%$ de mulheres, determinando uma razão homem/mulher de 4:1, que diminuiu ao longo do período de estudo, tendo passado de 5,2:1, em 1991, para 3,6:1, em 1995.

Em termos de idade, 2,2\% dos óbitos foram de crianças (menores 13 de anos), 0,8\% de adolescentes (13 a 19 anos), 84,3\% de adultos jovens (20 a 49 anos) e $12,7 \%$ de adultos com 50 anos ou mais. A estratificação por sexo mostrou que a distribuição etária foi semelhante para homens e mulheres, diferindo apenas na proporção de crianças, que foi maior no sexo feminino.
Nos indivíduos adultos, a média de idade dos homens foi de 37,8 anos e das mulheres de 37,3. A mediana das idades foi de 36 anos para os homens e 35 anos para as mulheres. Nos óbitos pelas demais causas, a idade média dos homens (56,5 anos), foi cerca de 19 anos mais elevada que nos óbitos por AIDS e nas mulheres $(66,4)$ cerca de 29 anos, indicando que, embora a AIDS provoque mortes prematuras em ambos os sexos, a transcendência destas mortes prematuras nas mulheres é ainda maior.

Nas crianças, a média de idade entre os óbitos por AIDS foi de 3,4 anos, dois anos a mais que nos óbitos pelas demais causas cuja média de idade entre os óbitos foi de 1,1 ano.

Em relação à escolaridade, o percentual de óbitos sem informação sobre o grau de instrução foi de $21,2 \%$ nos óbitos por AIDS, e 42,2\% nos óbitos pelas demais causas. Considerando apenas os óbitos com informação conhecida, observou-se que o percentual de indivíduos com nível universitário foi 2,5 vezes maior entre os óbitos por AIDS (14\%) que entre os óbitos pelas demais causas $(5,6 \%)$. Ao mesmo tempo, a proporção de indivíduos analfabetos foi 2,3 vezes menor entre os óbitos por AIDS (4,0\%) do que entre os óbitos pelas demais causas $(9,3 \%)$

Em relação ao estado civil, o percentual de óbitos sem informação foi de 3,1 \% nos óbitos por AIDS e de 5,7\%, nos óbitos pelas demais causas. Cerca de $75,0 \%$ dos indivíduos que morreram por AIDS eram solteiros, enquanto, nos óbitos pelas demais causas, esse percentual foi de 56,3\%. Da mesma forma, o percentual de casados nos óbitos pelas demais causas foi de $37,7 \%$ contra $18,9 \%$ nos óbitos por AIDS. A estratificação por sexo mostrou que essas relações se mantiveram para homens e mulheres no que se refere à proporção de casados e solteiros. Chamou a atenção, no entanto, que, entre as mulheres, a proporção de viúvas e divorciadas, respectivamente $10,0 \%$ e $5,1 \%$ entre os óbitos por AIDS, tenha sido quase o dobro da observada entre os óbitos pelas demais $(5,8 \% \mathrm{e}$ $2,8 \%$ ), enquanto, nos homens, as proporções mantiveram-se praticamente as mesmas.

A distribuição por área geográfica de residência mostrou grande concentração dos óbitos nas áreas metropolitanas, com a Microrregião do Rio de Janeiro concentrando $88 \%$ dos óbitos por AIDS, mas apenas $74 \%$ dos óbitos pelas demais causas. Focalizando apenas a capital do Estado, essa diferença foi ainda maior: o Município do Rio de Janeiro concentrou $64 \%$ dos óbitos por AIDS contra $44 \%$ dos óbitos pelas demais causas. Apesar disso, durante o período de estudo, a proporção dos óbitos por 
AIDS, nas áreas metropolitanas, diminuiu, principalmente na capital do Estado, tendo passado de 67,3\%, em 1991, para 61,6\%, em 1995.

\section{Mortalidade proporcional por AIDS}

No período de 1991 a 1995, a AIDS foi responsável por cerca de $2 \%$ do total de óbitos. Foi, todavia, na população entre 20 e 49 anos que se observaram-se os maiores percentuais de óbitos por AIDS (Figura 1).

No grupo de idade de 20 a 49 anos, a AIDS destacou-se entre as principais causas de morte. Excluindo-se as causas mal definidas, a mortalidade proporcional por AIDS cresceu, entre os homens, de $6,5 \%$, em 1991 , para $9,1 \%$, em 1995, e, entre as mulheres, de $3,1 \%$, para $6,9 \%$. Ainda que comparada com os grandes grupos de causa, agregados segundo os 17 capítulos da CID-9 (OMS, 1985), a AIDS ocupava, já em 1991, o terceiro lugar como causa de óbito entre os homens, superada apenas pelas causas externas e pelas doenças cardiovasculares, tendo essa posição sido mantida durante todo o período do estudo. Entre as mulheres, em 1991, a AIDS se constituiu na oitava causa de morte, passando a ocupar a quinta posição no ano de 1995. Para o último ano de estudo e também para os indivíduos de 20 a 49 anos, foram selecionadas, excluindo-se as afecções mal definidas, as dez primeiras causas de óbito agregadas segundo a lista de mortalidade da CID-9 (OMS, 1985) (Tabela 1).

Em ambos os sexos, a AIDS passa a constituir-se na segunda causa de morte. Nos ho- mens, apenas os homicídios responderam por mais mortes do que a AIDS e, nas mulheres, apenas as doenças cerebrovasculares. Considerando-se grupos etários com intervalos menores, observa-se que a AIDS é a principal causa de morte nas mulheres com idades entre 25 e 34 anos.

\section{Taxas de mortalidade especificas por AIDS}

No Estado do Rio de Janeiro, a mortalidade por AIDS passou de 11,8/100.000, em 1991, para 18,7/100.000, em 1995, correspondendo a um aumento de $58 \%$. A estratificação por sexo mostrou que esse crescimento foi observado tanto para homens $(20,6 / 100.000$ para $30,2 / 100.000)$ quanto para as mulheres $(3,7 / 100.000$ para $7,9 /$ 100.000), ainda que tenha sido menor para o sexo masculino ( $46 \%$ versus $113 \%$ ). Ressalta-se a sobremortalidade masculina, que passou de 5,6 em 1991 para 3,8, em 1995. A série histórica das taxas de mortalidade por AIDS nas décadas de 1980 e 1990 para homens e mulheres é apresentada na Figura 2.

Embora o aumento das taxas, inclusive nos anos mais recentes, observa-se que a curva de mortalidade por AIDS apresentou um crescimento acentuado até 1989 , período em que o crescimento das taxas ultrapassa $50 \%$ ao ano. Considerando-se homens e mulheres conjuntamente, a taxa de mortalidade por AIDS cresceu de 0,9/100.000 em 1986 para 2,7 em 1987, 4,6 em 1988 e 7,1 em 1989. A partir de então observa-se desaceleração na velocidade de

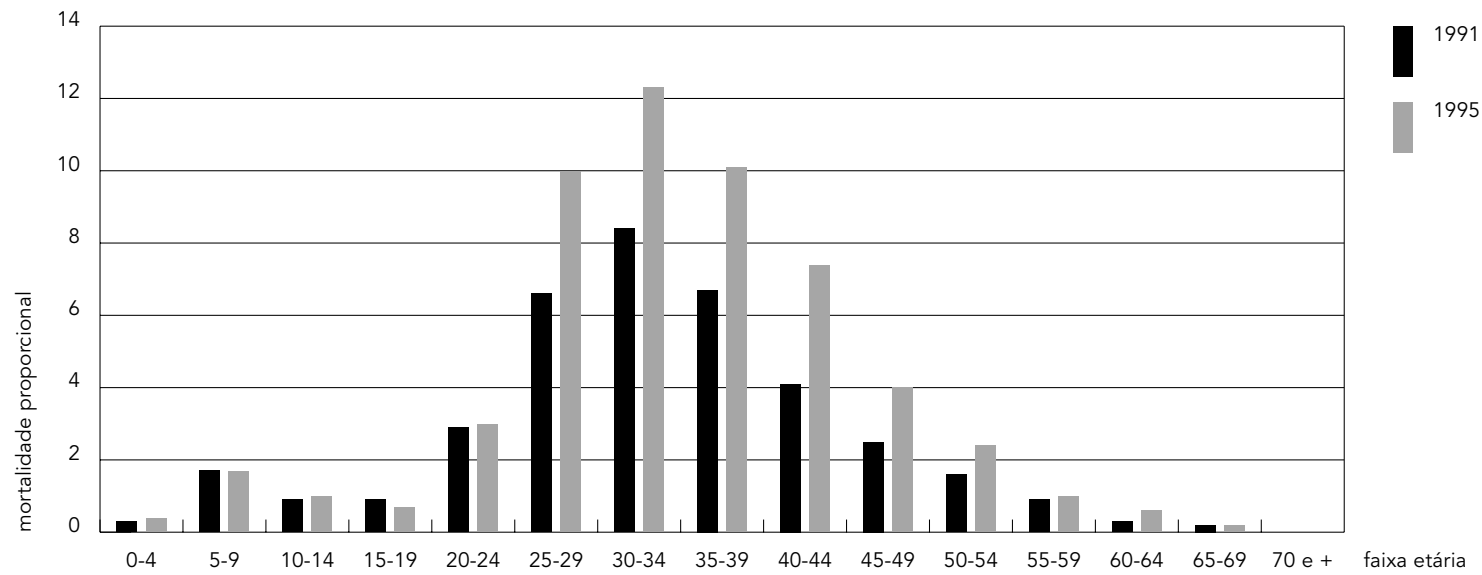


Tabela 1

Número de óbitos das dez primeiras causas de morte em homens e mulheres de 20 a 49 anos.

Estado do Rio de Janeiro, 1995.

\begin{tabular}{|c|c|c|c|c|c|c|c|}
\hline \multirow[t]{2}{*}{ Sexo/Causas } & \multirow[b]{2}{*}{$20-24$} & \multicolumn{5}{|c|}{ Faixas Etárias } & \multirow[t]{2}{*}{ Total } \\
\hline & & $25-29$ & $30-34$ & $35-39$ & $40-44$ & $45-49$ & \\
\hline \multicolumn{8}{|l|}{ Masculino } \\
\hline Homicídios (960-969) & 1.579 & 1.178 & 815 & 639 & 426 & 295 & 4.932 \\
\hline AIDS (279.1) & 63 & 276 & 404 & 393 & 314 & 173 & 1.623 \\
\hline $\begin{array}{l}\text { Acidente de trânsito por veículo a motor } \\
\text { (E810-E819) }\end{array}$ & 296 & 274 & 277 & 301 & 234 & 209 & 1.591 \\
\hline Infarto agudo do miocárdio (410) & 11 & 16 & 55 & 135 & 224 & 331 & 772 \\
\hline Doença cerebrovascular (430-438) & 20 & 32 & 61 & 112 & 241 & 302 & 768 \\
\hline Doenças crônicas do fígado e Cirrose (571) & 8 & 24 & 67 & 103 & 165 & 189 & 556 \\
\hline Tuberculose (010-018) & 19 & 45 & 75 & 108 & 125 & 107 & 479 \\
\hline Pneumonia (480-486) & 40 & 63 & 87 & 97 & 86 & 101 & 474 \\
\hline Quedas acidentais (E880-E888) & 44 & 38 & 37 & 72 & 61 & 40 & 292 \\
\hline Diabetes mellitus (250) & 5 & 16 & 28 & 55 & 75 & 100 & 279 \\
\hline Todas as causas $(001-999)^{\star}$ & 2.779 & 2.717 & 2.817 & 3.129 & 3.181 & 3.256 & 17.876 \\
\hline \multicolumn{8}{|l|}{ Feminino } \\
\hline Doença cerebrovascular (430-438) & 18 & 43 & 67 & 139 & 183 & 317 & 767 \\
\hline AIDS (279.1) & 43 & 83 & 96 & 96 & 77 & 56 & 451 \\
\hline Infarto agudo do miocárdio (410) & 6 & 6 & 30 & 75 & 115 & 146 & 378 \\
\hline Homicídios (960-969) & 70 & 68 & 71 & 54 & 43 & 23 & 329 \\
\hline Neoplasma maligno de mama (174) & 2 & 7 & 32 & 46 & 89 & 134 & 310 \\
\hline $\begin{array}{l}\text { Acidente de trânsito por veículo a motor } \\
\text { (E810-E819) }\end{array}$ & 59 & 45 & 56 & 49 & 52 & 47 & 308 \\
\hline Diabete mellitus (250) & 14 & 18 & 18 & 34 & 54 & 85 & 223 \\
\hline Doença hipertensiva (401-405) & 7 & 7 & 18 & 36 & 65 & 71 & 204 \\
\hline Pneumonia (480-486) & 13 & 23 & 38 & 43 & 36 & 42 & 195 \\
\hline Tuberculose (010-018) & 14 & 24 & 31 & 38 & 31 & 29 & 167 \\
\hline Todas as causas (001-999) & 533 & 644 & 881 & 1.203 & 1.470 & 1.766 & 6.497 \\
\hline
\end{tabular}

* Excluídas as causas mal definidas.

crescimento das taxas cujo crescimento de $34 \%$ observado entre $1989(7,1 / 100.00)$ e $1990(9,4 /$ 100.000 ) reduz-se a $8 \%$ nos dois últimos anos da série (17,3/100.000 em 1994 e 18,7 em 1995).

Em termos de idade, considerando-se todo o período de estudo ou cada ano em particular, observou-se que as faixas etárias entre 25 e 49 anos apresentaram as taxas de mortalidade mais elevadas tanto para os homens quanto para as mulheres (Figura 3). A comparação do primeiro e último ano do estudo, contudo, mostrou que, dos 20 aos 54 anos de idade, o crescimento das taxas foi maior entre as mulheres, ocorrendo o inverso a partir dos 55 anos. Verifica-se que, no grupo de 20 a 24 anos, enquanto a mortalidade masculina apresentou decréscimo (13,5/100.000 em 1991 para 10,8 em 1995), a mortalidade feminina teve seu maior crescimento (2,1/100.000 em 1991 para 7,1 em 1995). A sobremortalidade masculina, exceto nos me- nores de 15 anos, foi observada em todos os outros grupos etários.

Em termos geográficos, as taxas mais elevadas encontram-se nas áreas metropolitanas. Dos dez municípios com as maiores taxas de mortalidade por AIDS no Estado (taxa média para o período), sete são da Microrregião do Rio de Janeiro, um da Microrregião Serrana e dois da Microrregião de Lagos. Chama a atenção que municípios como Cabo Frio e Saquarema tenham respectivamente a quarta e sétima maiores taxas do Estado (Tabela 2).

A Figura 4 mostra as taxas de mortalidade por AIDS segundo município de residência, agregados em mesorregiões para três dos cinco anos de estudo (1991, 1993 e 1995).

A distribuição espacial das taxas mostrou que, no período estudado, o número de municípios sem óbitos por AIDS diminuiu, passando de 29, em 1991, para 21, em 1993, e 14, em 
1995. Ao mesmo tempo, o número de municípios com taxas entre 10 e 20/100.000 cresceu de 6, em 1991, para 14, em 1993 e 21, em 1995. Cabe ainda mencionar que, em 1991, não existiam municípios com taxas entre 20 e 30/ 100.000. Já em 1995, três municípios (Rio de Janeiro, Niterói e Nilópolis) apresentaram taxas maiores que 20/100.000.

\section{Anos Potenciais de Vida Perdidos}

No período de 1991 a 1995, houve, no Estado do Rio de Janeiro, um total de 7.985.721 APVP-65, dos quais $271.460(3,4 \%)$ foram devidos à AIDS. Nos homens, o percentual correspondente foi de $4,0 \%$ e, nas mulheres, de $2,2 \%$. Considerando os APVP-65 por todas as causas, a proporção referente aos homens foi duas vezes maior que a referente às mulheres $(67,1 \%$ e $32,9 \%$ respectivamente). Entre os APVP-65 devidos à AIDS, a proporção referente aos homens $(79,0 \%)$ foi cerca de quatro vezes maior que a referente às mulheres $(21,0 \%)$. Observando-se cada ano do estudo, verificou-se que a AIDS aumentou sua participação entre os APVP-65 em ambos os sexos, tendo passado, nos homens, de 3,4\%, em 1991 , para $4,7 \%$, em 1995 , e, nas mulheres, respectivamente de $1,4, \%$ para $2,9 \%$.

Os APVP-65 para a AIDS e agravos selecionados que se constituem em importantes causas de morte, são apresentados na Tabela 3.

Verifica-se que, entre os homens, no ano de 1991, o número de APVP-65 em conseqüência da AIDS era menor que os decorridos de cada um de todos os outros agravos selecionados, exceto do infarto agudo do miocárdio. Já em 1995, os APVP-65 devidos à AIDS superaram, além dos ocorridos pelo infarto, aqueles devidos às neoplasias e às doenças cerebrovasculares. Os APVP-65 pela AIDS passaram, nos homens, de 34.572, em 1991, para 51.073, em 1995,

\section{Figura 2}

Taxa de mortalidade (por 100.000 habitantes) por AIDS. Estado do Rio de Janeiro, 1984 a 1995

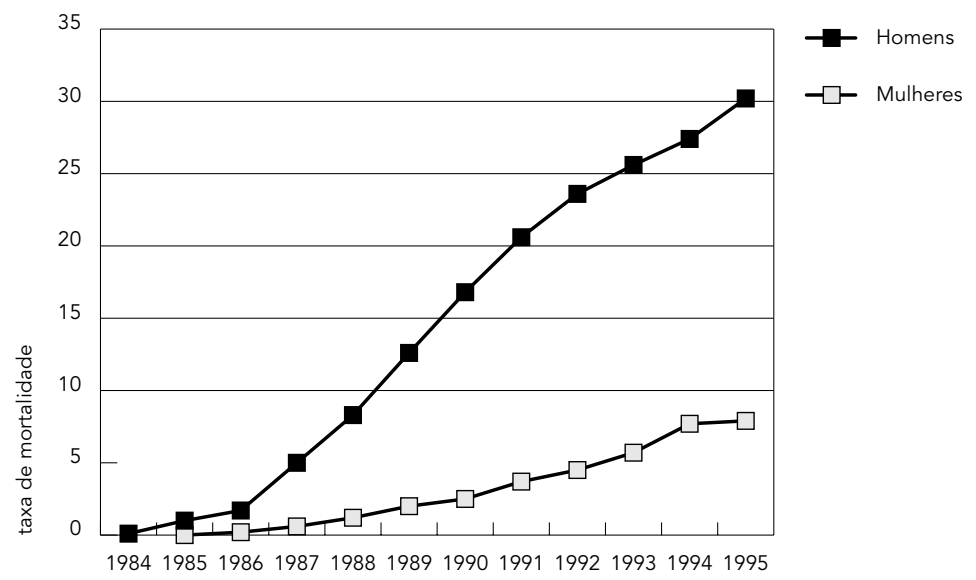

Taxa de mortalidade (por 100.000 habitantes) por AIDS segundo sexo e faixa etária. Estado do Rio de Janeiro, 1991 e 1995.

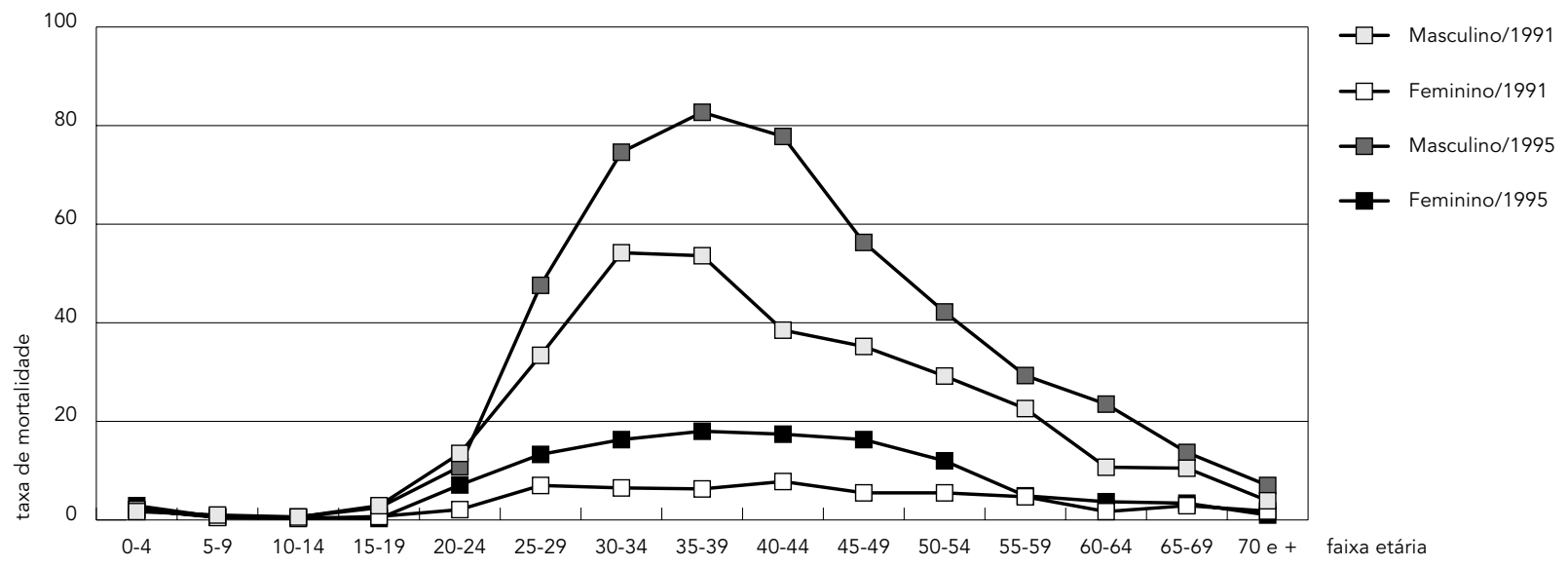


Tabela 2

Municípios com as dez maiores taxas de mortalidade por AIDS em ordem decrescente. Estado do Rio de Janeiro, 1991 a 1995.

\begin{tabular}{lll}
\hline Microrregião & Município & Mortalidade por AIDS (Taxas/100.000) \\
\hline Rio de Janeiro & Rio de Janeiro & 22,97 \\
Rio de Janeiro & Niterói & 20,14 \\
Rio de Janeiro & Nilópolis & 13,65 \\
Lagos & Cabo Frio & 13,12 \\
Serrana & Petrópolis & 12,55 \\
Rio de Janeiro & Duque de Caxias & 12,05 \\
Lagos & Saquarema & 11,17 \\
Rio de Janeiro & São João de Meriti & 10,90 \\
Rio de Janeiro & Nova Iguaçu & 10,65 \\
Rio de Janeiro & São Gonçalo & 10,41 \\
\hline
\end{tabular}

o que representou um crescimento de cerca de $48 \%$, superado apenas pelos homicídios que apresentaram, para o mesmo período, um crescimento de $60 \%$. Nas mulheres, a AIDS foi, em 1991, a última causa em termos de APVP65. No entanto, em 1995 os APVP-65 em decorrência da AIDS superaram os causados pelo infarto agudo do miocárdio e se aproximaram do número de APVP-65 devido aos homicídios. O número de APVP-65 devidos à AIDS apresentaram, nas mulheres, um crescimento acentuado, passando de 6.759, em 1991, para 15.544, em 1995, o que corresponde a um aumento de cerca de $130 \%$.

\section{Discussão e conclusão}

Entre os achados da presente análise, o primeiro aspecto que merece comentário é: embora os resultados tenham revelado o crescimento das taxas de mortalidade específicas por AIDS, revelaram também uma diminuição na velocidade desse crescimento. Esse dado é compatível com o que vem sendo observado nos Estados Unidos que, em 1996, apresentaram pela primeira vez uma diminuição em números absolutos dos óbitos por AIDS (CDC, 1996a) e com os dados do Município de São Paulo que também mostraram queda no número de óbitos por AIDS no ano de 1996 (SMS/SP, 1997). Nos Estados Unidos, a queda na mortalidade é atribuída à diminuição da incidência da doença associada à melhoria do tratamento (CDC, 1996b). No Brasil, mesmo que venha sendo observado declínio na velocidade de crescimento da epidemia em estados como São Paulo (Moraes et al., 1997), em função de diversos fato- res, entre eles o atraso das notificações, ainda é cedo para se falar em diminuição da incidência da doença. A hipótese principal é que, pelo menos em grande parte, a diminuição da mortalidade por AIDS seja conseqüência do aumento da sobrevida dos pacientes devido à melhoria das condições de tratamento, especialmente à terapêutica combinada de antiretrovirais associada à profilaxia das infecções oportunistas. As expectativas são que, com os inibidores de protease, disponíveis no Brasil desde de dezembro de 1996, observe-se queda ainda maior na mortalidade. Tanto nos Estados Unidos quanto em São Paulo, a queda no total de óbitos se deu em função da diminuição dos óbitos masculinos, ainda que tenha sido observada desaceleração no crescimento dos óbitos femininos. Este é um dado que também vai ao encontro do verificado no presente trabalho, visto que no período estudado a taxa de mortalidade cresceu, entre homens, $46 \%$, e nas mulheres, $113 \%$.

Apesar de apresentar uma tendência de declínio, a AIDS causou no Estado do Rio de Janeiro, nos cinco anos de estudo, impacto na mortalidade, mormente entre os adultos jovens (20-49 anos). Nesta faixa etária, a AIDS aumentou sua participação relativa no total de óbitos, passando a constituir-se em uma das principais causas de morte. No último ano do estudo, a AIDS respondeu por uma maior proporção de óbitos do que o infarto agudo do miocárdio, diabetes ou acidentes de trânsito, sendo superada, nos homens, apenas pelos homicídios e, nas mulheres, apenas pelas doenças cerebrovasculares. No Município de São Paulo, entre 1991 e 1996, resultados semelhantes foram encontrados para a faixa etária de 15 a 49 anos, na qual a AIDS foi, nos homens, também superada apenas pelos homicídios, e, nas mulheres, ela se constitui na principal causa de morte desde 1993 (SMS/SP, 1997).

$\mathrm{O}$ fato de as mais altas taxas de morte apresentarem-se entre os adultos jovens está em consonância com dados de morbi-mortalidade nacionais (Ferreira \& Castiñeiras, 1996; Fundação SEADE, 1997; MS, 1997a) e internacionais (Bouvier-Colle et al., 1994; CDC, 1996b; Regidor et al., 1995; Valdespino-Gómez et al., 1995). Também a sobremortalidade masculina é um fato conhecido devido à maior incidência da doença entre os homens. Em São Paulo, a sobremortalidade masculina devido à AIDS e às causas externas vem sendo inclusive apontada como um dos determinantes da ampliação da diferença entre a esperança de vida feminina e masculina (Ferreira \& Castiñeiras, 1996). Contudo, deve-se ressaltar que, no Estado do Rio 
de Janeiro, a sobremortalidade masculina vem caindo na faixa etária de 20 a 49 anos, o que reflete a maior velocidade de crescimento da mortalidade por AIDS entre as mulheres desse grupo de idade.

Outro aspecto que vale ser ressaltado refere-se ao maior crescimento das taxas de morte por AIDS entre mulheres mais jovens (particularmente dos 20 aos 25 anos de idade) e entre homens mais velhos (acima de 55 anos), o que poderia estar relacionado com comportamentos usuais na busca da parceria sexual, em que homens tendem a procurar mulheres mais jovens, o que, pelo menos em parte, faria com que as mulheres se infectassem em idades mais precoces do que os homens.

O impacto da mortalidade por AIDS no adulto jovem pode ainda ser traduzido pela idade média de morte observada (37 anos) e pelos APVP em decorrência da doença, em que, principalmente entre as mulheres, evidencia-se um crescimento bastante expressivo (130\%). A ascensão do número de mortes prematuras - expressão última da relevância da epidemia - qualifica e reitera sua transcendência. Atingindo segmentos da população em idade produtiva/reprodutiva, a AIDS, em especial nos países em desenvolvimento, agrava a desagregação familiar e a orfandade. No Brasil, de acordo com dados do Projeto Mundial para Órfãos (Comitê Assessor do Projeto Mundial para Órfãos, 1996), estima-se que, até junho de 1996, dez mil e seiscentas crianças com menos de 14 anos tenham perdido suas mães em decorrência da AIDS e doenças associadas. Estes fenômenos, embora antigos, em curso com a epidemia, assumem novas dimensões e vêm gerando custos econômicos e sociais imensuráveis. Ressalte-se ainda que, na ausência de qualquer intervenção terapêutica, a mediana do período de latência clínica da doença é de onze anos (Rachid \& Schechter, 1996) e, de acordo com Bartlett (1996) o tempo médio decorrido entre a imunossupressão grave que caracteriza a AIDS (CD4 abaixo de 200células $/ \mathrm{mm}^{3}$ ) e o óbito é de aproximadamente 24 a 36 meses. Assim, se a mortalidade por AIDS tem seu maior impacto na população entre 20 e 49 anos é possível inferir que a infecção pelo HIV venha ocorrendo em grupos etários ainda mais jovens.

Em termos geográficos, mesmo sendo preponderante a importância da AIDS como causa de morte nas áreas metropolitanas, observou-se, ao longo do período estudado, a diminuição proporcional dos óbitos por AIDS na Microrregião do Rio de Janeiro, e em particular na capital do Estado, assim como o aumento
Figura 4

Taxa de mortalidade por AIDS segundo municípios agregados por mesorregiões. Estado do Rio de Janeiro, 1991, 1993 e 1995.
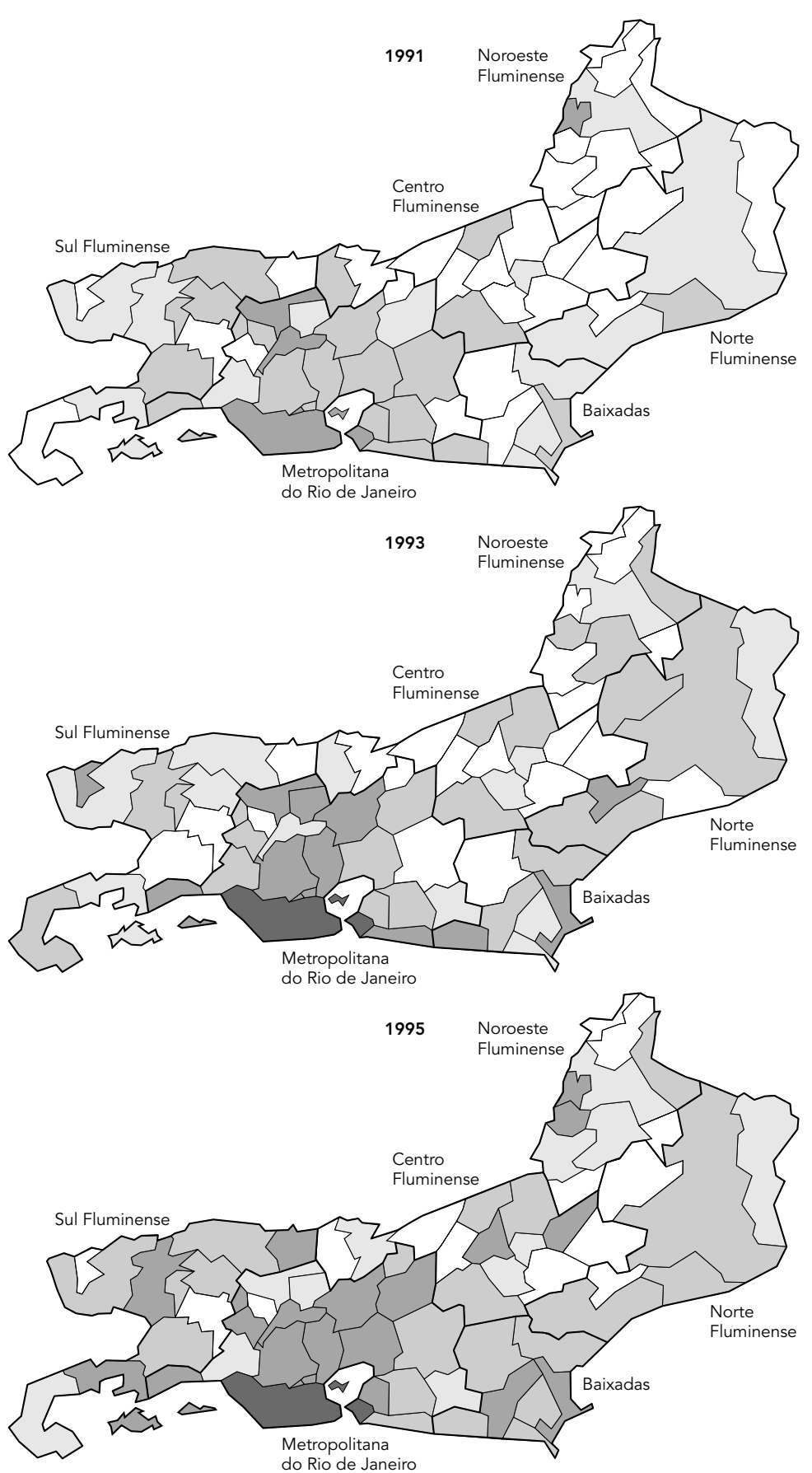

Taxa de mortalidade por AIDS (100.000 hab)
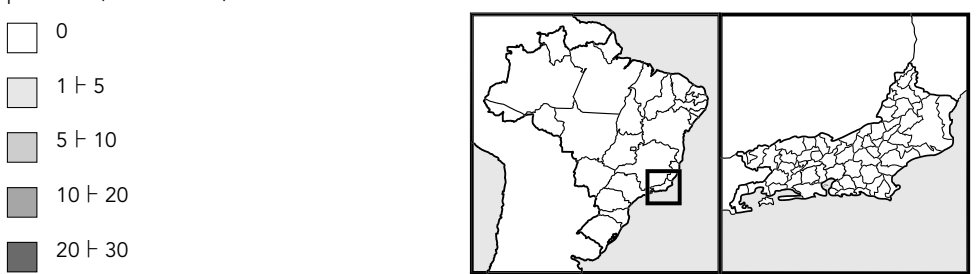
Tabela 3

Anos potenciais de vida perdidos por AIDS e causas selecionadas.

Estado do Rio de Janeiro, 1991 a 1995.

\begin{tabular}{lrrrr}
\hline Causas & \multicolumn{2}{c}{1991} & \multicolumn{2}{c}{1995} \\
& Homem & Mulher & Homem & Mulher \\
\hline AIDS (279-1) & 34.572 & 6.759 & 51.073 & 15.544 \\
DIP & 58.577 & 37.571 & 61.375 & 37.492 \\
Neoplasias & 43.584 & 44.732 & 45.807 & 49.132 \\
Homicídios & 144.666 & 10.881 & 231.630 & 16.519 \\
Acidentes de trânsito & 54.195 & 15.275 & 69.519 & 19.609 \\
por veículos a motor & & & & \\
Infarto agudo do miocárdio & 31.105 & 14.445 & 28.983 & 14.075 \\
Doenças cerebrovasculares & 35.048 & 28.204 & 30.274 & 28.347 \\
Todas as causas & 1.018 .274 & 498.920 & 1.096 .235 & 535.380 \\
\hline
\end{tabular}

do número de municípios de outras regiões que apresentaram taxas de morte por AIDS, o que sugere a disseminação da epidemia do cinturão metropolitano para municípios do interior do Estado. Vale ainda destacar os resultados obtidos para municípios como Cabo Frio e Saquarema pertencentes à Microrregião de Lagos que apresentaram taxas de morte por AIDS elevadas e crescentes, o que provavelmente associa-se ao fato de ser esta uma região turística, com uma população flutuante nos finais de semana e durante o verão, favorecendo uma maior densidade demográfica e de interação social, fatores que, segundo Barcellos \& Bastos (1996), cumprem papel importante na difusão da epidemia.

Na comparação do perfil dos óbitos por AIDS com aquele obtido para as demais causas, destacam-se as diferenças observadas em relação à escolaridade e estado civil. No que tange à escolaridade, embora os dados de morbidade do Estado do Rio de Janeiro indiquem que o nível de instrução dos indivíduos notificados venha caindo ao longo dos anos (SES/RJ, 1996) e dados referentes ao Brasil e Município de São Paulo apontem para um aumento das taxas de incidência e deslocamento da concentração dos óbitos para áreas mais empobrecidas (Bastos et al., 1995; Drumond Jr. et al., 1996/1997; UNAIDS, 1997), os resultados do presente estudo mostraram que o nível de escolaridade entre os indivíduos que morreram de AIDS foi mais elevado que o verificado para os óbitos pelas demais causas. Em relação ao estado civil, observaram-se uma maior proporção de solteiros e uma menor proporção de casados entre os indivíduos que faleceram de AIDS. Entre as mulheres chamou a atenção o fato de haver entre os óbitos por AIDS uma proporção de viúvas e divorciadas quase duas vezes maior que nos óbitos pelas demais causas, enquanto nos homens, não houve diferença. Uma das hipóteses é que as viúvas, tanto quanto as divorciadas e solteiras, teriam um maior número de parceiros sexuais que as casadas e, portanto, maior chance de infecção. Porém, é possível também aventar a hipótese de que as mulheres viúvas já teriam perdido seus companheiros pela AIDS, principalmente se levarmos em conta que mulheres casadas (condição anterior das viúvas) tendem à parceria única, adotando usualmente comportamentos associados à percepção equivocada de que não estão expostas a risco.

Por último, cabe enfatizar que o presente trabalho sofre as limitações comuns advindas da utilização de dados de mortalidade que têm sua qualidade afetada por diversos fatores tais como o acesso diferenciado aos serviços de saúde, dificuldades no diagnóstico, erro no preenchimento da declaração de óbito, na codificação da causa básica e até no processamento dos dados. Esses fatores afetam, de forma diferenciada, os estudos de mortalidade por causa específica e no caso da AIDS, pelo fato de ser uma doença associada a estigmas e preconceitos, a preocupação de sua subestimação como causa de morte é referida por vários autores (Barchielli et al., 1995; Buchalla, 1993; Gold et al., 1994; Montellá-i-Jordana et al., 1995).

Outro aspecto a ser levantado diz respeito à codificação da causa básica. A categoria 279.1, referente à deficiência da imunidade celular, CID-9 (OMS, 1985), é anterior ao aparecimento da AIDS e agrupa, ainda, outras três síndromes que, no entanto, são afecções bastante raras e cujos indivíduos acometidos dificilmente sobrevivem além da infância ou adolescência (Buckley, 1996).

Em relação à população, as estimativas referentes aos anos de 1992 a 1995 foram calculadas tendo como base a tendência de crescimento do período intercensitário de 1980 a 1991 e, de acordo com a contagem populacional realizada em 1996, estão superestimadas. Assim, tanto a possível omissão da AIDS como causa de morte quanto as estimativas populacionais tendem a enviesar o presente estudo no sentido de subestimar as taxas de mortalidade específica por AIDS e os indicadores de mortalidade proporcional por esta causa de morte.

Em conclusão, os resultados mostram que a mortalidade por AIDS apresenta desaceleração na velocidade de crescimento, notadamente entre os homens. Ainda assim, constitui-se 
em importante causa de morte, atingindo principalmente uma população jovem, sexual e economicamente ativa, ocasionando desta forma muitas mortes prematuras. Levando-se em conta que o óbito é um evento tardio, os dados indicam que a infecção pelo HIV vem ocorrendo em faixas etárias ainda mais precoces, sugerindo que as medidas de prevenção sejam direcionadas para adolescentes e adultos jovens.

\section{Referências}

BARCELLOS, C. \& BASTOS, F. I., 1996. Redes sociais e difusão da AIDS no Brasil. Boletín de la Oficina Sanitaria Panamericana, 121:11-24.

BARCHIELLI, A.; BUIATTI, E.; GALANTI, C.; GIOVANNETTI, L.; ACCIAI, S. \& LAZZERI, V., 1995. Completeness of AIDS reporting and quality of AIDS death certification in Tuscany (Italy): A linkage study between surveillance system of cases and death certificates. European Journal of Epidemiology, 11:513-517.

BARTLETT, J. G., 1996. Tratamento Clínico da Infecção pelo HIV. São Paulo: Editora Três.

BASTOS, F. I.; TELLES, P. R.; CASTILHO, E. \& BARCELLOS, C., 1995. A epidemia da AIDS no Brasil. In: Os Muitos Brasis: Saúde e População na Década de 80 (M. C. S. Minayo, org.), pp. 245-268, São Paulo: Editora Hucitec/Rio de Janeiro: ABRASCO.

BINDELS, P. J. E.; REIJNEVELD, S. A.; MULDERFOLKERTS, D. K. F.; COUTINHO, R. A. \& HOEK, A. A. R., 1994. Impact of AIDS on premature mortality in Amsterdam, 1982-1992. AIDS, 8:233-237.

BOUVIER-COLLE, M. H.; JOUGLA, E. \& SCHWOEBEL, V., 1994. Impact du SIDA sur la mortalité générale en France. Revue d'Epidémiologie et Santé Publique, 42:89-98.

BUCHALLA, C. M., 1993. A Síndrome da Imunodeficiência Adquirida e a Mortalidade Masculina, de 20 a 49 Anos, no Município de São Paulo: 1983 a 1986. Tese de Doutorado, São Paulo: Faculdade de Saúde Pública, Universidade de São Paulo.

BUCKLEY, R. H., 1996. Primary immunodeficiency diseases. In: Cecil Textbook of Medicine (C. Bevett \& F. Plum, ed.), pp. 1401-1408, 20th Ed. Philadelphia: W. B. Saunders Company.

CDC (Centers for Disease Control and Prevention), 1996a. 1996 HIV/AIDS Trends Provide Evidence of Success in HIV Prevention and Treatment: AIDS Deaths Decline for First Time. February 1997 $<$ http://www.cdc.gov>.

CDC (Centers for Disease Control and Prevention), 1996b. Update: Mortality attributable to HIV infection among persons Aged 25-44 years - United States, 1994. MMWR, 45:121-143.

CDC (Centers for Disease Control and Prevention) / WHO (World Health Organization), 1996. Epi Info 6, Version 6.04. A Word Processing, Database, and Statistics Program for Public Health. Atlanta: CDC/ Geneva: WHO.
COMITÊ ASSESSOR DO PROJETO MUNDIAL PARA ÓRFÃOS. 1996. Projeto Mundial para Órfãos. 17 Março 1998 <www.jsi.com/brasil/gop/gop00001. htm>.

DRUMOND Jr., M.; LIRA, M. M. T. A.; FREITAS, M. \& NITRINI, T. M. V., 1996/1997. A AIDS e os sistemas de informações de mortalidade em nível local: A experiência do PRO-AIM no Município de São Paulo. AIDS Boletim Epidemiológico, 9:3-9.

FERREIRA, C. E. \& CASTIÑEIRAS, L. L., 1996. O rápido aumento da mortalidade dos jovens adultos em São Paulo: Uma trágica tendência. São Paulo em Perspectiva, 10:1-9.

FUNDAÇÃO SEADE (Fundação Sistema Estadual de Análise de Dados), 1997. Taxa de Mortalidade por AIDS, por Sexo e Faixa Etária: Município de São Paulo 1988-1995. 19 setembro 1997 <http://www. seade.gov.br>.

GOLD, J.; YUEMING, L. \& KALDOR, J. M., 1994. Premature mortality in Australia 1983-1992, the first decade of the AIDS epidemic. Medical Journal of Australia, 161:652-656.

KUZMA, J. W., 1984. Basic Statistics for the Health Sciences. Mountain View: Mayfield Publications.

LA VECCHIA, C.; FRANCESCHI, S.; DAL MASO, L. \& PARAZZINI, F., 1994. Impact of the AIDS epidemic on mortality of young men in Italy. Journal of Acquired Immune Deficiency Syndromes, 7:873874.

MAPINFO CORPORATION, 1994. Map Info. New York: MapInfo Corporation.

MONTELLÁ-I-JORDANA, N.; RICART-DE-MESONES, I.; BORRELL-I-THIÓ, C.; CLOS-I-GUIX, R. \& CAYLÁ-IBUQUERAS, J., 1995. Comparación de las defunciones del registro de casos de sida y de las defunciones por sida del registro de mortalidad Barcelona; 1991-1992. Revista de Sanidade e Higiene Pública, 69:49-57.

MORAES, J. C.; SEABRA, N. S. S. \& ELUF NETO, J., 1997. São Paulo. In: A Epidemia da AIDS no Brasil: Situação e Tendências. Brasília: Ministério da Saúde.

MS (Ministério da Saúde), 1997a. AIDS. Boletim Epidemiológico, 9(6).

MS (Ministério da Saúde), 1997b. Sistema de Informação sobre Mortalidade - 1979 a 1996. CD-ROM. Brasília: MS.

MUR, C. B.; MAYANS, M. V.; MOMPART PENINA, A.; ALBARRACIN, G. P. \& BARBARA, J. C., 1995. Im- 
pacto del sida en la mortalidad global de Cataluña, 1981-1993. Medicina Clinica, 105:528-531.

OMS (Organização Mundial de Saúde), 1985. Manual da Classificação Estatística Internacional de Doenças, Lesões e Causa de Óbito - Nona Conferência de Revisão. São Paulo: Centro Brasileiro de Classificação de Doenças em Português.

PERRE, P. V., 1995. The epidemiology of HIV infection and AIDS in Africa. Trends in Microbiology, 3:217222.

RACHID, M. \& SCHECHTER, M., 1996. Manual de HIV/ AIDS. Rio de Janeiro: Livraria e Editora Revinter Ltda.

REGIDOR, E.; BARRIO, G.; LA FUENTE, L. \& RODRIGUEZ, C., 1995. Impacto de la infección por el virus de la inmunodeficiencia humana en la mortalidad de los jóvenes en España. Medicina Clinica, 105:515.

SELIK, R. M.; CHU, S. Y. \& BUEHLER, J. W., 1993. HIV infection as leading cause of death among young adults in US cities and states. JAMA, 269:29912994.
SES-RJ (Secretaria de Estado de Saúde - Rio de Janeiro), 1996. Sistema de Informação de Agravos de Notificação - SINAN. Dados Registrados até 31/12/ 1996. Rio de Janeiro: SES/RJ.

SMS-SP (Secretaria Municipal da Saúde - São Paulo), 1997. Programa de DST/AIDS. A mortalidade por AIDS. Boletim Epidemiológico, 1:1-16.

UNAIDS (Joint United Nations Programme on HIV/AIDS), 1997. Report on the Global HIVIAIDS Epidemic. 17 February 1998 <www.unaids.org/ mgnband/document/report97.html .

VALDESPINO-GOMEZ, J. L.; GARCIA-GARCIA, M. L.; DEL RIO-ZOLEZZI, A.; LOO-MENDEZ, E.; MAGISRODRIGUEZ, C. \& SALCEDO-ALVAREZ, R. A., 1995. Epidemiología del SIDA/VIH en México: De 1983 a marzo de 1995. Salud Pública de México, 37:556-571.

WALDVOGEL, B., 1994. Monitoramento das mortes por AIDS: Uma vigilância necessária. Conjuntura Demográfica, 27/28:13-19. 